\title{
Primary Cutaneous Leiomyosarcoma of the Lower Extremity: A Case Report and Literature Review
}

\author{
Victor Chalfant ${ }^{1}$, Tyler Schriber ${ }^{1}$, Ahmed Sabri ${ }^{1}$, John Gossen ${ }^{1}$, Darren Groh ${ }^{1}$ \\ 1. Department of Pathology, Creighton University School of Medicine, Omaha, USA
}

Corresponding author: Victor Chalfant, victorchalfantmd@gmail.com

\begin{abstract}
Cutaneous leiomyosarcoma is a rare soft-tissue sarcoma that appears non-specific clinically and often is misdiagnosed as squamous cell carcinoma. We report the case of a 59-year-old Caucasian male with a grade I leiomyosarcoma tumor on his lower extremity with no previous history of local trauma. The tumor is composed of highly atypical spindle cells with pleomorphic nuclei and mitotic activity on hematoxylin and eosin stains. The diagnosis is confirmed with immunohistochemistry staining positive for smooth muscle actin, vimentin, and desmin. Due to high recurrence rates, the prognosis for leiomyosarcomas remains poor and requires close follow-up to prevent progression.
\end{abstract}

Categories: Dermatology, Pathology, Oncology

Keywords: neoplasm recurrence, sarcoma soft tissue, cutaneous leiomyosarcoma, mohs surgery, wide local excision, h\&e staining, desmin, vimentin, smooth-muscle actin, dermatopathology

\section{Introduction}

Cutaneous leiomyosarcoma is a rare neoplasm, with an annual incidence of 0.2 per 100,000, commonly occurring in the sixth and seventh decades of life. These tumors are most commonly found on the extremities, and also are found often on the trunk, or head and neck, presenting as asymptomatic nodules that may or may not have erythema or tenderness [1]. The two types of leiomyosarcomas are leiomyosarcomas arising from the arrector pili muscles and subcutaneous leiomyosarcomas arising from the adipose tissue's vascular smooth muscle. Because of this, subcutaneous leiomyosarcomas are usually larger, staged higher, and subsequently more aggressive. For either subtype, the prognosis is worse when the larger tumor is diagnosed [2]. A biopsy is necessary for the proper diagnosis of cutaneous leiomyosarcomas, as well as distinguishing the true depth of the lesion. Immunohistochemistry will show vimentin and smooth muscle actin in all cases while showing muscle actin and desmin in over half of cases [3]. After diagnosis, treatment is most often wide local excision or rarely Mohs micrographic surgery. The use of adjuvant chemotherapy and immunotherapy is still controversial, and an area of continued study, but is the normal course when resection is not possible $[1,4]$. There has been shown to be a sizable recurrence rate, greater than $24 \%$, for both subtypes, though the malignancy rate for the subcutaneous subtype is much higher, which tends to need closer follow-up [5]. Here we present the rare case of a cutaneous leiomyosarcoma presenting symptomatically, without metastasis, with a focus on efficient diagnosis and surgical treatment.

Review began 03/26/2021 Review ended 04/02/2021 Published 04/03/2021

\section{() Copyright 2021}

Chalfant et al. This is an open access article distributed under the terms of the Creative Commons Attribution License CC-BY 4.0., which permits unrestricted use, distribution, and reproduction in any medium, provided the original author and source are credited.

\section{Case Presentation}

A 59-year-old Caucasian man with a past medical history of chronic obstructive pulmonary disease is referred by his primary care physician for a suspicious skin lesion measuring $1.4 \mathrm{~cm}$ on his right anterolateral thigh due to concern of it increasing in size over the past year and starting to become significantly tender (Figure 1). The patient noticed the lesion over a year ago. The patient reports the use of $325 \mathrm{mg}$ tablets of acetaminophen every six hours as needed for pain. The patient denies taking any medication regularly. The patient denies any associated fatigue, unexplained weight loss, bone pain, fever, nausea/vomiting, or changes in his bowel/bladder habits. The patient reports no past history of intense sun exposure or history of skin cancer. In social history, the patient denies usage of alcohol, however, reports an 88-pack-year of smoking. The patient has no family history of cancer in first-degree relatives. The area around the lesion is anesthetized with $1 \mathrm{ml}$ of $2 \%$ xylocaine with epinephrine. A subsequent $8 \mathrm{~mm}$ shave biopsy of the lesion is taken by an electrodessication and curettage (ED\&C) technique. The biopsy is placed in buffered formalin and sent for biopsy. Antibiotic ointment is applied and a sterile dressing placed. 


\section{Cureus}

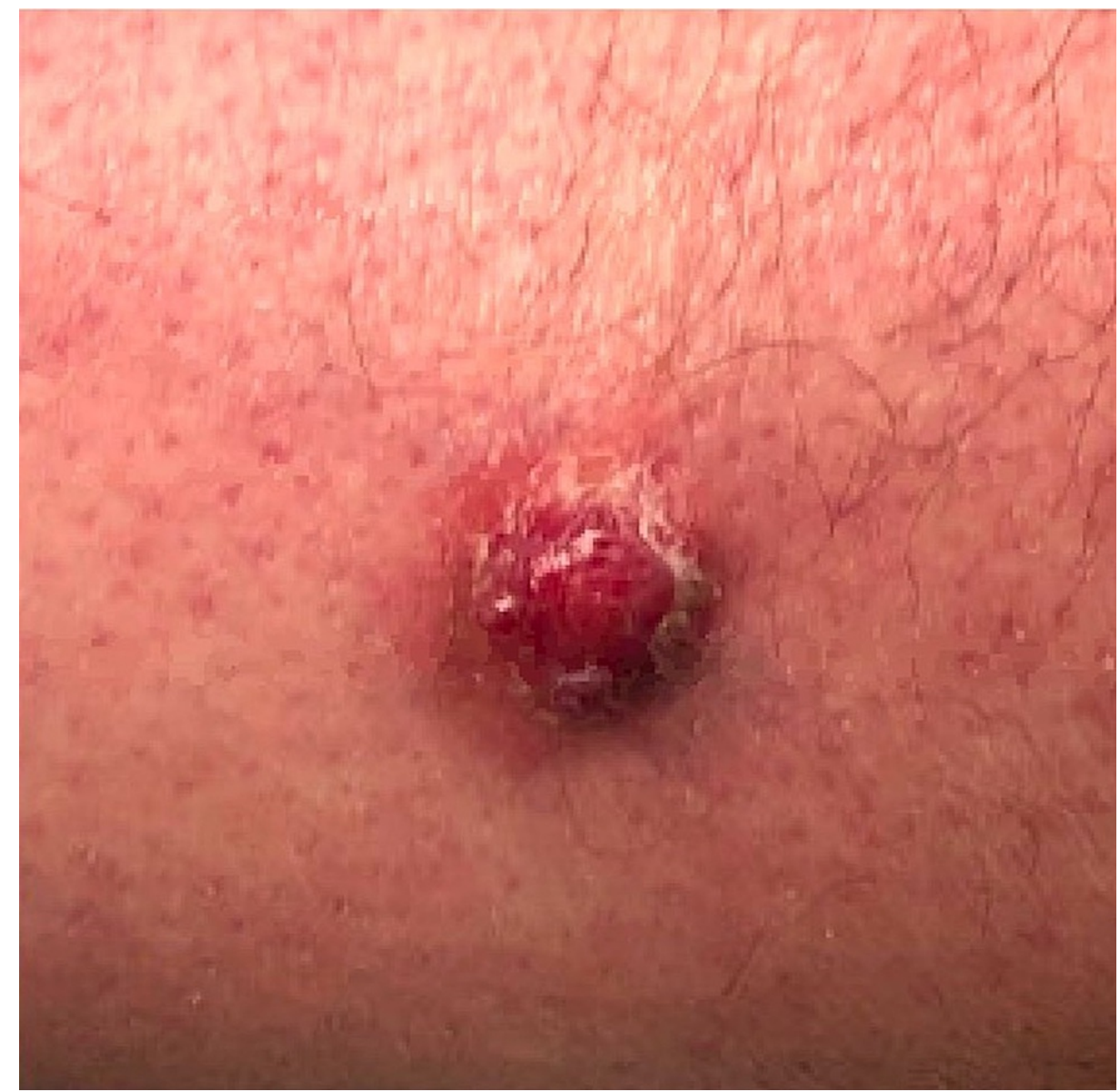

FIGURE 1: $1.4 \mathrm{~cm}$ purplish eroded lesion on the right anterior thigh.

Laboratory investigations are significant for a hemoglobin of 17.8 grams per decilitre and a white blood count of 6,000 per cubic millimeter of blood. The section shows highly atypical spindle cells with pleomorphic nuclei and mitotic activity on hematoxylin and eosin stains (Figures $2 A, 2 B$ ). The biopsy section extends from the epidermis to the deep dermis (Figure 2C). The tumor cells are arranged in long and short fascicles (Figures $3 A, 3 B$ ). Positive staining is present for smooth muscle actin, vimentin, and desmin immunostains (Figures $4 A-4 C, 5 A, 5 B$ ). No staining is observed for cytokeratin AE1/3, p63, SOX10, S100, HMB45, CD68, CD10, or CD31. Based on the Fédération Nationale des Centres de Lutte Contre Le Cancer, histology supports a grade I leiomyosarcoma due to the presence of a well-differentiated tumor with a low mitotic rate (six mitoses per 10 high-power fields) and an absence of necrosis [6].

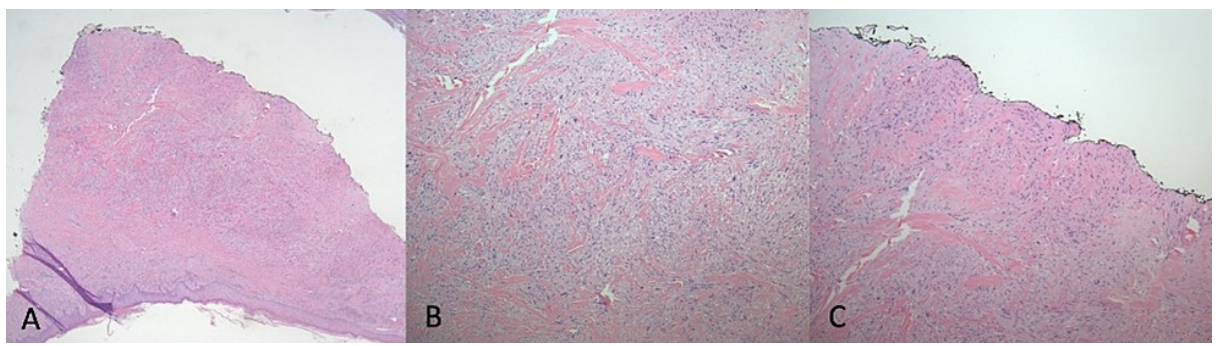

FIGURE 2: Hematoxylin and eosin (H\&E) stains under low power view at $4 x(A), 10 x(B)$, and of the biopsy site showing the tumor extending into the deep margin at $10 x(C)$. 


\section{Cureus}

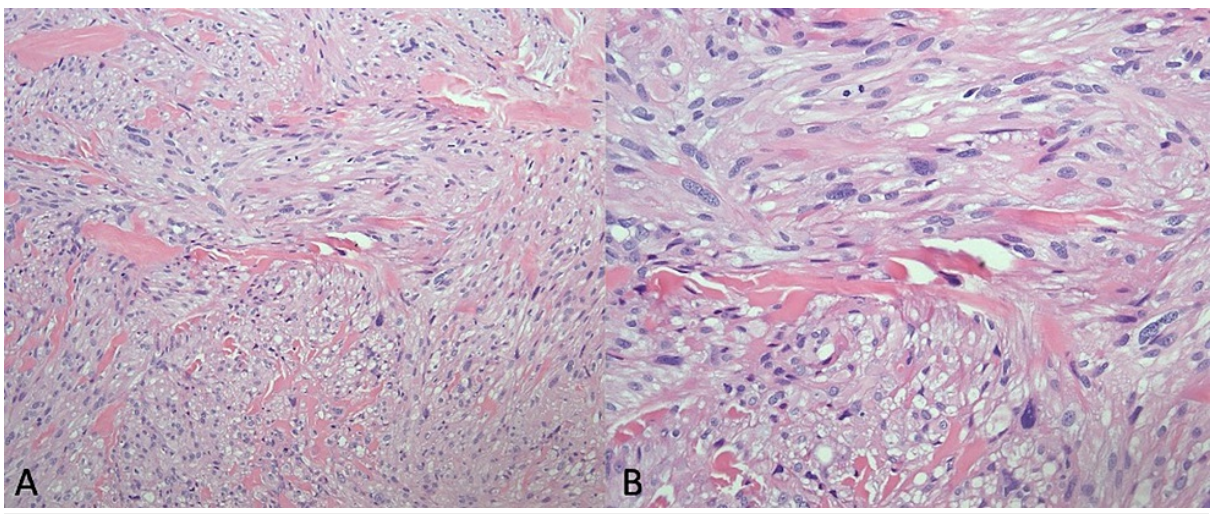

FIGURE 3: High power view at 20x (A) and 40x (B) showing highly atypical spindle cells with pleomorphic nuclei and mitotic activity.

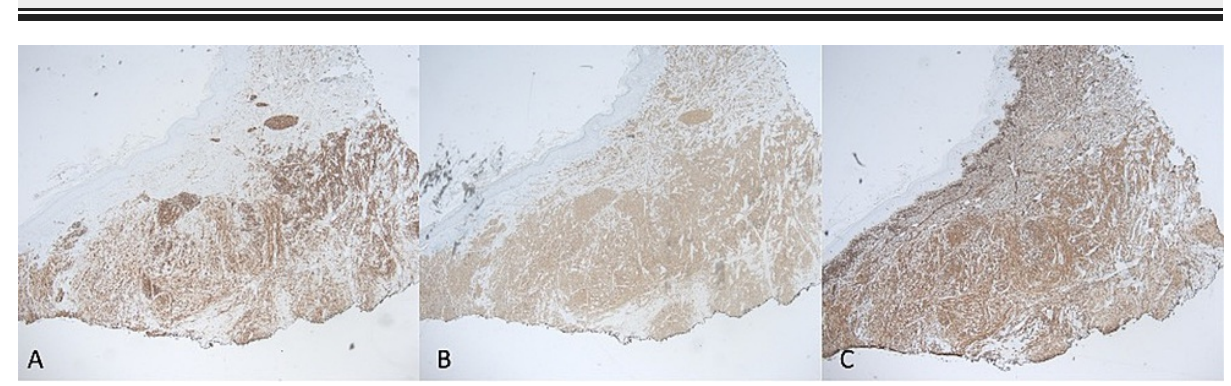

FIGURE 4: Immunostains show positive staining for desmin (A), smooth muscle actin (B), and vimentin (C) under low power view at $4 x$.

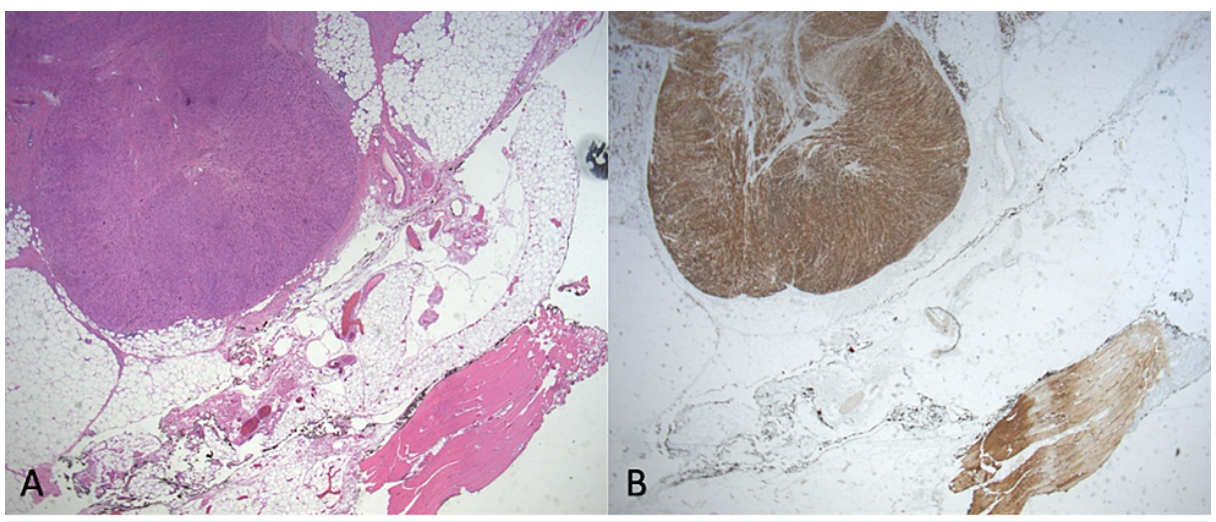

FIGURE 5: H\&E stain (A) and immunostain of desmin (B) under low power view at $10 x$ of the resection site.

Right femur MRI with contrast shows enhancing skin lesion in the anterolateral distal thigh extending into the subcutaneous soft tissue with skin thickening measuring 2.6 × 1.4 × $3.0 \mathrm{~cm}$ (Figures 6A, 6B). Nonspecific right inguinal lymph nodes were also noted on imaging. In order to assess the possibility of metastatic disease, CT imaging studies of the chest, abdomen, and pelvis were ordered. Chest CT scan with contrast is significant for a $5.6 \mathrm{~mm}$ non-calcified left upper lobe pulmonary nodule. Pulmonary function tests determine the functional expiratory volume (FEV1) of $47 \%$. 


\section{Cureus}

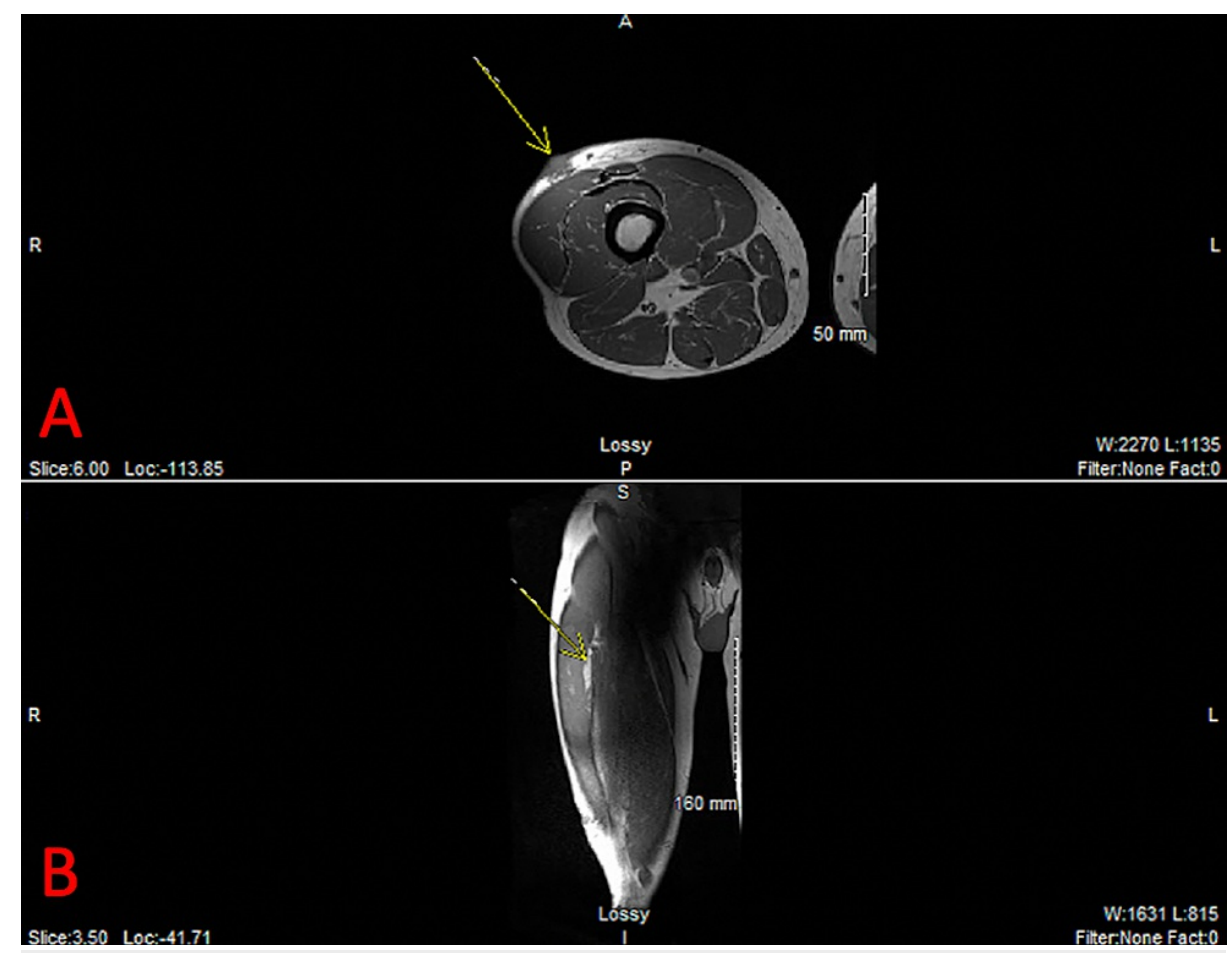

FIGURE 6: Enhancing lesion in the right anterolateral distal thigh with associated skin thickening and extension of the subcutaneous soft tissue in transverse dimension, marked by yellow arrow (A).

Enhancement extends along the superficial fascial margin of the vastus lateralis muscle without definitive muscle invasion in the frontal plane, marked by yellow arrow (B).

The patient is treated with wide local excision of the right lower extremity under general anesthesia. An ulcerated tan-yellow-gray circumscribed mass with skin ellipse measuring $5 \mathrm{~cm} \times 4.2 \mathrm{~cm} \times 2 \mathrm{~cm}$ is obtained. Pathology confirms negative margins with $1.7 \mathrm{~cm}$ from the medial margin, $1.9 \mathrm{~cm}$ from the caudal margin, and $0.5 \mathrm{~cm}$ from the deep margins. No lymph nodes are identified.

At one-week follow up, the incision appears to be healing well with a small amount of sanguineous drainage. The wound is redressed in the office with an elastic bandage. The patient reports having ceased smoking since the procedure. At subsequent one-month follow-up, the incision appears dry with no erythema. Sutures are removed and dressing changed at an office visit. The patient is scheduled for follow-up over the next two years quarterly.

\section{Discussion}

Primary cutaneous leiomyosarcoma is a rare soft tissue sarcoma that is believed to occur due to the malignant transformation of arrector pili muscles in the dermis. While cutaneous leiomyosarcoma is typically present at a lower stage with a five-year disease-specific survival of $98 \%$, high-grade leiomyosarcoma can see a drop in survival to $50 \%$ after five years and requires more immediate treatment [7]. On presentation, patients typically complain of increasing pain associated with the sudden growth of the mass along with other findings such as ulceration, paresthesia, and bleeding. According to our knowledge, over the last 10 years, there are 11 other reported cases in the literature based on a PubMed search (Table 1). Of note, many of the cases report a previous history of local trauma, chronic non-healing ulcer, or prior leiomyosarcoma. 


\begin{tabular}{|c|c|c|c|c|c|c|c|c|c|c|}
\hline Year & Sex & Onset Age & History & Site & Size & Grade & Treatment & Margins & Recurrence & Reference \\
\hline 2014 & M & 79 & Local Trauma & Anterior Trunk & $4 \mathrm{~cm}$ & 1 & WLE & $2 \mathrm{~cm}$ & 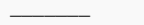 & [1] \\
\hline 2013 & $\mathrm{M}$ & 70 & Chronic Ulcer & Lower Extremity & $5 \mathrm{~cm}$ & III & WLE & $5 \mathrm{~cm}$ & - & [2] \\
\hline 2018 & M & 60 & Chronic Ulcer & Lower Extremity & $9 \mathrm{~cm}$ & $\longrightarrow$ & WLE & $\longrightarrow$ & - & [8] \\
\hline 2018 & M & 30 & None & Anterior Trunk & $5 \mathrm{~cm}$ & III & WLE & $1 \mathrm{~cm}$ & 72 months & [9] \\
\hline 2015 & M & 27 & Previous LMS & Lower Extremity & $7 \mathrm{~cm}$ & II & WLE & $1 \mathrm{~cm}$ & 24 months & [10] \\
\hline 2018 & $\mathrm{M}$ & 59 & None & Upper Extremity & $1 \mathrm{~cm}$ & {[} & WLE & - & - & [11] \\
\hline 2018 & W & 32 & LFS & Upper Extremity & $2 \mathrm{~cm}$ & 1 & WLE & - & 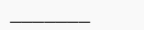 & [12] \\
\hline 2011 & M & 74 & None & Posterior Trunk & $3 \mathrm{~cm}$ & 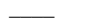 & WLE & - & - & [13] \\
\hline 2018 & $\mathrm{~F}$ & 54 & Local Trauma & Face & $5 \mathrm{~cm}$ & III & WLE & - & 12 months & [14] \\
\hline 2012 & M & 66 & Local Trauma & Scapula & - & 1 & WLE & $1 \mathrm{~cm}$ & 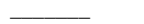 & [15] \\
\hline 2021 & $\mathrm{~F}$ & 93 & Previous LMS & Upper Extremity & $11 \mathrm{~cm}$ & III & Amputation & - & 36 months & [16] \\
\hline 2021 & M & 59 & None & Lower Extremity & $1 \mathrm{~cm}$ & 1 & WLE & $1 \mathrm{~cm}$ & & \\
\hline
\end{tabular}

TABLE 1: A literature review of recent cutaneous leiomyosarcoma case reports.

Abbreviations: LMS, leiomyosarcoma; WLE, wide-local excision; LFS, Li-Fraumeni syndrome.

As the typical presentation of leiomyosarcoma is nonspecific clinically, definitive diagnosis depends on a histopathological skin biopsy. On H\&E stain, the atypical spindle-shaped cells with cigar-shaped nuclei and eosinophilic cytoplasm are often arranged in poorly circumscribed fascicles. Typically, more than two mitoses per 10 high-power fields are identified. Immunohistochemistry of vimentin and smooth muscle actin is present in all cases, while desmin is present in over half of cases [3]. S-100 immunostaining has rarely been observed [17]. Kaddu et al. have described two primary histopathological growth patterns: nodular or diffuse [3]. Nodular is characterized by high cellularity, lots of mitotic figures, and the presence of necrosis whereas diffuse is characterized by low cellularity, few mitotic figures, and the absence of necrosis [3].

Cutaneous leiomyosarcoma is primarily treated with wide local excision, preferably with $1 \mathrm{~cm}$ margins in order to promote disease-free survival. In a review of 112 cases, Aneiros-Fernandez et al. found rates of recurrence to be $36.63 \%$ after an average follow-up time of 4.40 years [5]. Increasingly Mohs surgery for leiomyosarcoma has increased in popularity, due to closer margin control and lower recurrence rates. Murphy-Chutorian et al. report recurrence rates of $2.08 \%$ to $6.25 \%$ compared to $30 \%$ to $50 \%$ with wide local excision [18]. Mohs surgery has not been widely adopted due to few long-term prognostication differences, particularly for deeper lesions. Both radiotherapy and chemotherapy seldom are incorporated as adjuvant modalities. Due to inadequate studies, the role of adjuvant therapy has remained anecdotal, with the usage of either chemotherapy or radiation reserved primarily for distant metastases or recurrence [4]. Doxorubicin, ifosfamide, gemcitabine, or taxotere are effective in controlling progression, however, they are not effective at curing metastatic disease [19].

Due to high levels of recurrence of cutaneous leiomyosarcoma, regular follow-up is necessary. There is currently no clear standard for aftercare. However, Massi et al. recommend a follow-up quarterly for the first two years followed by a biannual visit for three years, and finally an annual visit for the next 20 years [20]. Radiography for possibly recurrence and metastases are included as part of a regular workup.

\section{Limitations}

Due to short follow-up, our study is limited in scope. Molecular studies were not performed as part of the workup for our case.

\section{Conclusions}

An enlarging and tender mass should raise suspicion for cutaneous leiomyosarcoma with histopathological workup required to rule out the diagnosis. Although Mohs microsurgery or the addition of adjuvant therapy has become increasingly used, limited studies are available to merit its use over wide local excision alone. Follow-up, however, should remain close to prevent malignant disease progression. 


\section{Additional Information \\ Disclosures}

Human subjects: Consent was obtained or waived by all participants in this study. Conflicts of interest: In compliance with the ICMJE uniform disclosure form, all authors declare the following: Payment/services info: All authors have declared that no financial support was received from any organization for the submitted work. Financial relationships: All authors have declared that they have no financial relationships at present or within the previous three years with any organizations that might have an interest in the submitted work. Other relationships: All authors have declared that there are no other relationships or activities that could appear to have influenced the submitted work.

\section{References}

1. Ciurea ME, Georgescu CV, Radu CC, et al.: Cutaneous leiomyosarcoma - Case report. J Med Life. 2014, $15: 270-3$.

2. Bali A, Kangle R, Roy M, et al.: Primary cutaneous leiomyosarcoma: a rare malignant neoplasm . Indian Dermatol Online J. 2013, 4:188-90. 10.4103/2229-5178.115513

3. Kaddu S, Beham A, Cerroni L, Humer-Fuchs U, Salmhofer W, Kerl H, Soyer HP: Cutaneous leiomyosarcoma. Am J Surg Pathol. 1997, 21:979-87. 10.1097/00000478-199709000-00001

4. Deneve JL, Messina JL, Bui MM, et al.: Cutaneous leiomyosarcoma: treatment and outcomes with a standardized margin of resection. Cancer Control. 2013, 20:307-12. 10.1177/107327481302000408

5. Aneiros-Fernandez J, Antonio Retamero J, Husein-Elahmed H, et al.: Primary cutaneous and subcutaneous leiomyosarcomas: evolution and prognostic factors. Eur J Dermatol. 2016, 26:9-12. 10.1684/ejd.2015.2681

6. Coindre JM, Terrier P, Guillou L, et al.: Predictive value of grade for metastasis development in the main histologic types of adult soft tissue sarcomas: a study of 1240 patients from the French Federation of Cancer Centers Sarcoma Group. Cancer. 2001, 14291:10-1914. 10.1002/1097-0142(20010515)91:10<1914::aidcncr1214>3.0.co;2-3

7. Workman AD, Farquhar DR, Brody RM, et al.: Leiomyosarcoma of the head and neck: a 17-year single institution experience and review of the National Cancer Data Base. Head Neck. 2018, 40:756-62. 10.1002/hed.25054

8. Ahluwalia VV, Singh PK, Singh H, et al.: Advanced magnetic resonance imaging in the evaluation of a chronic non-healing ulcer: a case report of rare primary cutaneous leiomyosarcoma. J Orthop Case Rep. 2018, 8:7-10. 10.13107/jocr.2250-0685.1134

9. Hmida L, Letaief F, Doghri R, et al.: Cutaneous leiomyosarcoma on the trunk: an unusual presentation with an aggressive course - Case report and review of literature. Pan Afr Med J. 2018, 31:190-10.

10. Abbasi F, Mahmudlu R, Nikniaz Y, et al.: Primary cutaneous leiomyosarcoma in a young patient previously misdiagnosed as pleomorphic fibroma. Iran J Pathol. 2015, 10:69-73.

11. Ortins-Pina A, Soares-de-Almeida L, Rütten A: Primary cutaneous vascular leiomyosarcoma: a rare subtype of leiomyosarcoma of the skin. J Cutan Pathol. 2018, 45:639-41. 10.1111/cup.13271

12. Sabater-Marco V, Ferrando-Roca F, Morera-Faet A, et al.: Primary cutaneous leiomyosarcoma arising in a patient with Li-Fraumeni syndrome: a neoplasm with unusual histopathologic features and loss of heterozygosity at TP53 gene. Am J Dermatopathol. 2018, 40:225-7. 10.1097/DAD.0000000000000919

13. Yamada S, Guo X, Yoshizawa M, et al.: Primary desmoplastic cutaneous leiomyosarcoma associated with high MIB-1 labeling index: a teaching case giving rise to diagnostic difficulties on a small biopsy specimen. Pathol Res Pract. 2011, 15:728-32. 10.1016/j.prp.2011.08.008

14. Murback NDN, Takita LC, Castro BC, et al.: Cutaneous leiomyosarcoma on the face. An Bras Dermatol. 2018, 93:262-4. 10.1590/abd1806-4841.20186715

15. Pol RA, Dannenberg H, Robertus JL, et al.: Cutaneous leiomyosarcoma arising in a smallpox scar. World J Surg Oncol. 2012, 16:148. 10.1186/1477-7819-10-14

16. Cazzato G, Sergi MC, Sablone S, et al.: Advanced cutaneous leiomyosarcoma of the forearm . Dermatopathology (Basel). 2021, 27:40-4. 10.3390/dermatopathology8010008

17. Kim NG, Kim JO, Park YJ, Kim JS, Lee YJ, Lee KS: Cutaneous leiomyosarcoma of the face. Arch Craniofac Surg. 2017, 18:145-8. 10.7181/acfs.2017.18.2.145

18. Murphy-Chutorian B, Routt E, Vinelli G, et al.: A systematic review of the treatment of superficial leiomyosarcoma with Mohs micrographic surgery. Dermatol Surg. 2019, 45:1437-41. 10.1097/DSS.0000000000001992

19. Garcia del Muro X, de Alava E, Artigas V, et al.: Spanish Group for Research on Sarcoma. Clinical practice guidelines for the diagnosis and treatment of patients with soft tissue sarcoma by the Spanish group for research in sarcomas (GEIS). Cancer Chemother Pharmacol. 2016, 77:133-46. 10.1007/s00280-015-2809-5

20. Massi D, Franchi A, Alos L, et al.: Primary cutaneous leiomyosarcoma: clinicopathological analysis of 36 cases. Histopathology. 2010, 56:251-62. 10.1111/j.1365-2559.2009.03471.x 